\title{
IN MEMORY \\ OF VYACHESLAV E. KUNITSYN
}

PACS 01.60.+q

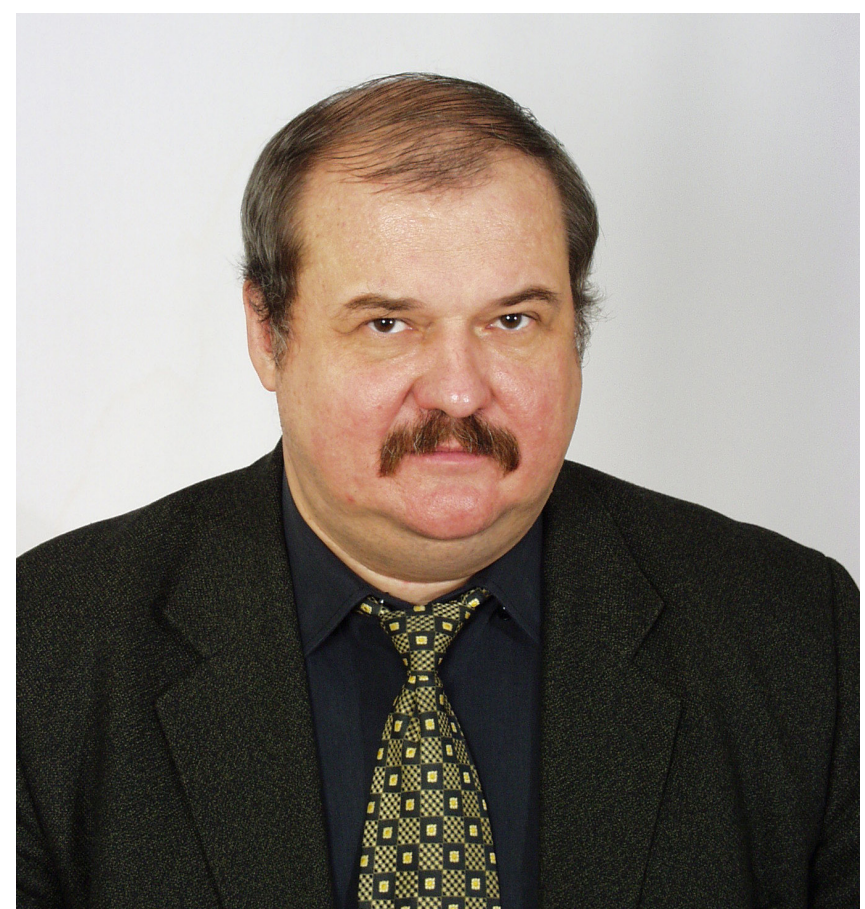

Outstanding Russian scientist, Academician of the Russian Academy of Natural Sciences and the International Academy of Higher School, Doctor of Physical and Mathematical Sciences, Head of Geophysics Division and Atmospheric Physics Chair of Physical Faculty of Lomonosov Moscow State University, professor Vyacheslav Evgenyevich Kunitsyn suddenly died on July 7, 2015 in his 61th year of life.

Kunitsyn Vyacheslav Evgenyevich was born June 24, 1955 in Gorky (now - Nizhny Novgorod). His father - Kunitsyn Evgeny Ivanovich was the military officer of the Soviet Army. His mother Kunitsyna Nadezhda Stepanovna was employee. In 1972 Vyacheslav Evgenyevich entered the Faculty of Physics of Lomonosov MSU. He graduated it with honors in 1978. In 1981 after completion of postgraduate study of MSU physics faculty he defended the master's thesis "The inverse problems of the theory of waves and restoration of scattering heterogeneity's structure with the degree in "radiophysics". During his study, the great influence on the formation of his scientific interests and future activities had been given by such outstanding scientists and outstanding personalities as R.V. Khokhlov, O.V. Rudenko, Y.L. Klimontovich, V.D.
DOI: $10.17725 /$ rensit.2015.07.100

Gusev, and A.N. Matveev. All life of V. E. Kunitsyn was associated with physics faculty of Lomonosov Moscow State University. Since 1981 Vyacheslav Evgenyevich worked as the assistant, the senior teacher, the associate professor, professor of MSU physics faculty. In 1991 he defended the doctoral dissertation "Radio tomography and radio sounding of an ionosphere" (specialty "radiophysics"). In 1994, he heads the chair of atmosphere physics, having become the youngest head of chair in MSU physics faculty.

The main directions of Vyacheslav Evgenyevich's academic researchers were problems of remote sounding of geophysical environments and objects by different types of waves, direct and inverse problems of wave theory, radio tomography of ionosphere and near space, physics of the upper atmosphere and ionosphere. Kunitsyn, V.E. developed methods for solving inverse problems of remote sensing of the atmosphere and near-earth environment. New solutions of one-dimensional inverse problems of layered medium sensing were got. The methods, for calculating the radio-wave propagation in the ionosphere and the atmosphere, taking into account wave effects (quantum tunneling and above-barrier reflection), allowed us to explain a number of phenomena, observed when probing by ionospheric recorders (wide-range transparency of sporadic layers, the resonance of group delay, etc.), were developed.

Over recent years, under the leadership of Vyacheslav Evgenyevich, methods, for satellite radio tomography of an ionosphere, including radial, diffractive and statistical radio tomography, were developed. On the basis of the developed methods, jointly with the Polar geophysical Institute Russian Academy of Sciences, the first experiments on RT ionosphere were conducted. World's first images of localized ionosphere irregularities (diffractive RT) were received, radio imaging contour intervals of the main ionospheric trough (radial RT) were reconstructed, the spatial distribution of electrondensity fluctuations (statistical RT) were obtained. He became a pioneer and a prominent 
leader in the development and application of radio imaging methods in geophysics, atmosphere physics and near space. Now it is difficult to imagine progress in physics of the higher atmosphere without original and significant scientific results, obtained by him and his students. V.E. Kunitsyn has attained honored reputation as a world-class specialist in the field of inospheric physics and upper atmosphere. He worked closely both with colleagues from different national academic and industrial institutions and with colleagues abroad. Very often the collaboration, which began with a joint work on projects, then, developed into close friendship.

Innovative, original radiophysical methods of ionosphere research, developed in 90-e years under the guidance of V.E. Kunitsyn, provided he achievement of results on the wide range of phenomena for many years ahead, actively studied at present time. Vyacheslav Evgenyevich had extraordinary intuition, relying on a deep and omnifarious knowledge. He readily shared his ideas with colleagues and rejoiced when they developed them. Besides knowledge in its immediate academic interest, Vyacheslav Evgenyevich was characterized by knowledge and erudition in many areas of physics - from the methods of digital processing of radio signals of modern navigation systems in studies of the upper atmosphere and near space to the deep radio sounding of the Martian polar ices using space vehicles and regional monitoring of the ionosphere by GPS interferometry. He aroused his colleagues' and students' interest in the novel scientific achievements. For over 20 years, V. E. Kunitsyn was giving courses, covering a wide range of issues - from introduction to atmospheric physics to relationships nd interactions in the atmosphere-ionosphere and remote sensing of the upper atmosphere.

Under the leadership of Vyacheslav Evgenyevich, the department staff together with a number of national and foreign scientific centers conducted a numerous RT researches in Russia, USA, Scandinavia, and Southeast Asia. The developed methods of satellite RT open prospect of creation regional and global monitoring systems of the atmosphere and near-earth space. For development of ionosphere satellite radio tomography method, V. E. Kunitsyn, as a part of development team of MSU staff, PIP of Russian Academy of Sciences and IZMIRAN n 1998, was awarded the State award of Russian Federation in the field of science and technology.

Vyacheslav Evgenyevich had published more than 400 works, including 4 monographs and 2 textbooks, had made numerous reports at the international and Russian conferences. Number of V. E. Kunitsyn's articles citations according to Web of Science is 562, Scopus -391.

Vyacheslav Evgenyevich was a member of Academic Council of MSU physics faculty, Chairman of the Academic Council of geophysics department, Chairman of the Dissertation Council of Moscow state University D.001.63 and a member of the Dissertation Council of EIA RAS, a member of Bureau for meteorology and atmospheric sciences of the national geophysical Committee, a member of the Bureau for radio wave propagation of RAS, a editorial Board member of the journal "Bulletin of Moscow University (Ser. Physics, Astronomy)" and "RENSIT - Radio Electronics, NanoSystems, and Information Technologies", a member of the Organizing Committee of the international Baikal fundamental physics school. Being a laureate of the State prize of the Russian Federation, Vyacheslav Evgenyevich was also the first laureate of Moscow State University Prize named after I. I. Shuvalov (1993) and Lomonosov Prize laureate (2005). He was an Academician of the Russian Academy of Natural Sciences (2000) and the International Academy of Sciences of Higher School (1996).

Among his students there were 16 candidates of Sciences. For successful research and teaching activities and active social work V. E. Kunitsyn was awarded medals "For labor valor" and "In honour of 850th anniversary of Moscow", badge of honor "250 years of Lomonosov Moscow State University".

Cherished memory of Vyacheslav Evgenyevich Kunitsyn - the outstanding scientist, talented teacher, solid friend will be preserved in the hearts of all those, who fortunate to know close, work together, and just chat with this brilliant scientist and a wonderful person.

Staff of Atmospheric Physics Chair of Lomonosov MSU Physics Faculty

Department of Radioelectronics, Nanophysics and Information Technologies' problems of Russian Academy of Natural Sciences Editorial Board of RENSIT 